Homology, Homotopy and Applications, vol. 22(1), 2020, pp.1-10

\title{
THE EULER CHARACTERISTIC OF THE REGULAR SPHERICAL POLYGON SPACES
}

\author{
YASUHIKO KAMIYAMA
}

(communicated by R. James Milgram)

\begin{abstract}
Let $a$ be a real number satisfying $0<a<\pi$. We denote by $M_{n}(a)$ the configuration space of regular spherical $n$-gons with side-lengths $a$. The purpose of this paper is to determine $\chi\left(M_{n}(a)\right)$ for all $a$ and odd $n$. To do so, we construct a manifold $X_{n}$ and a function $\mu: X_{n} \rightarrow \mathbb{R}$ such that $\mu^{-1}(a)=M_{n}(a)$. In fact, the function $\mu$ is different from the Kapovich-Millson Morse function. We determine the index of each critical point of $\mu$. Since a level set is obtained by successive Morse surgeries, we can determine $\chi\left(M_{n}(a)\right)$.
\end{abstract}

\section{Introduction and statement of the main result}

Recently, the topology of polygon spaces in the Euclidean space of dimension two or three has been considered by many authors. The study of planar polygon spaces started in $[\mathbf{4}, \mathbf{8}, \mathbf{1 5}]$. For example, the homology groups were determined in $[\mathbf{2}]$. On the other hand, the study of spatial polygon spaces started in $[\mathbf{9}]$.

Morse theory plays a key role in the study of polygon spaces. [1] is an excellent exposition about polygon spaces with emphasis on Morse theory. In [12], Milgram and Trinkle obtained results by making excellent use of Morse surgery.

Later, Kapovich and Millson [11] studied spherical polygon spaces. They proved a remarkable and very useful theorem about a Morse function. We first recall their result.

Let $r=\left(r_{1}, r_{2}, \ldots, r_{n}\right)$ be an $n$-tuple of real numbers satisfying $0<r_{i}<\pi$. We set $r^{\prime}:=\left(r_{1}, \ldots, r_{n-1}\right)$ and define

$$
\widetilde{N}_{r^{\prime}}=\left\{u=\left(u_{1}, \ldots, u_{n}\right) \in\left(S^{2}\right)^{n} \mid d\left(u_{i}, u_{i+1}\right)=r_{i}, 1 \leqslant i \leqslant n-1\right\} .
$$

Here $d$ is the spherical distance. Let $S O(3)$ act on $\widetilde{N}_{r^{\prime}}$ diagonally and we set $N_{r^{\prime}}:=$ $\widetilde{N}_{r^{\prime}} / S O(3)$. Kapovich and Millson defined the function $\rho_{n}: N_{r^{\prime}} \rightarrow \mathbb{R}$ by

$$
\rho_{n}(u)=d\left(u_{1}, u_{n}\right) .
$$

We will restrict to $u$ 's such that $0<\rho_{n}(u)<\pi$ so that $\rho_{n}$ is differentiable. Note that

$$
M_{r}:=\rho_{n}^{-1}\left(r_{n}\right) \subset N_{r^{\prime}}
$$

is the moduli space of closed polygonal linkages in $S^{2}$ with side-lengths $\left(r_{1}, \ldots, r_{n}\right)$.

Received March 14, 2018, revised April 6, 2019; published on September 18, 2019.

2010 Mathematics Subject Classification: 58E05, 58D29.

Key words and phrases: spherical polygon space, Morse function, Euler characteristic.

Article available at http://dx.doi.org/10.4310/HHA.2020.v22.n1.a1

Copyright (C) 2019, International Press. Permission to copy for private use granted. 
It is proved in [11] that an element of $N_{r^{\prime}}$ is a critical point of $\rho_{n}$ if and only if it is degenerate, i.e., it lies in a great circle $\gamma$ in $S^{2}$. In order to describe the signature, we give the following:

Definition 1.1 (orienting $\gamma$ ). Suppose $u=\left(u_{1}, u_{2}, \ldots, u_{n}\right)$ is a closed degenerate linkage contained in a great circle $\gamma$. Orient $\gamma$ so that the arc joining $u_{1}$ to $u_{n}$ is positively directed. Thus an edge $e_{i}$ is a back-track if it has the same direction as $e_{n}=\left(u_{n}, u_{1}\right)$.

Theorem $1.2([\mathbf{1 1}$, Main Theorem $])$. Let $T \in N_{r^{\prime}}$ be a degenerate free $(n-1)$ linkage and $P$ be the associated degenerate closed $n$-gon linkage. Then the signature of $\left.D^{2} \rho_{n}\right|_{T}$ is given by

$$
(b(P)+2 w(P)-1, f(P)-2 w(P)-1) .
$$

Here $f=f(P)$ denotes the number of forward-tracks and $b=b(P)$ denotes the number of back-tracks so $f+b=n$. Moreover, $w(P)$ denotes the winding number of the degenerate configuration $P$, which is given by the formula $\sum_{i=1}^{n} \varepsilon_{i} r_{i}=2 \pi w(P)$, where $\varepsilon_{i} \in\{ \pm 1\}$ is defined to be 1 or -1 according as $e_{i}$ is forward-track or $e_{i}$ is back-track.

Let $a$ be a real number satisfying $0<a<\pi$. We consider the case that $r=$ $(a, a, \ldots, a)$, that is, $r_{i}=a$ for $1 \leqslant i \leqslant n$. We set

$$
M_{n}(a):=\rho_{n}^{-1}(a)
$$

Note that $M_{n}(a)$ is the moduli space of regular spherical polygons with side lengths $a$.

When $n$ is even, $M_{n}(a)$ has critical points for all $a$. More precisely, singular points are given by the degenerate polygons. Hence, in what follows, we will always assume $n$ to be odd and set $n=2 m+1$.

The purpose to this paper is to determine $\chi\left(M_{n}(a)\right)$. For that purpose, we realize $M_{n}(a)$ as a level set of a Morse function, which is different from the above $\rho_{n}$. We first set

$$
\begin{aligned}
& A_{n}:=\left\{(P, a)=\left(\left(u_{1}, \ldots, u_{n}\right), a\right) \in\left(S^{2}\right)^{n} \times(0, \pi) \mid d\left(u_{i}, u_{i+1}\right)=a \text { for } 1 \leqslant i \leqslant n-1\right. \\
& \text { and } \left.d\left(u_{n}, u_{1}\right)=a\right\} \text {. }
\end{aligned}
$$

Let $S O(3)$ act on $A_{n}$ by

$$
(P, a) g:=(P g, a)
$$

where $g \in S O(3)$. Then we set

$$
X_{n}:=A_{n} / S O(3)
$$

We define the function $\mu: X_{n} \rightarrow \mathbb{R}$ by

$$
\mu(P, a)=a .
$$

Note that for all $a \in(0, \pi)$, we have

$$
\mu^{-1}(a)=M_{n}(a) .
$$

The following Theorems 1.3 and 1.4, which are main theorems of this paper, assert that $\mu$ is a Morse function. Since a level set is obtained by successive Morse surgeries, we can determine $\chi\left(M_{n}(a)\right)$ for all $a$. 
Theorem 1.3. An element $(P, a) \in X_{n}$ is a critical point of $\mu$ if and only if $P$ is degenerate.

Thus the critical points of $\rho_{n}$ and $\mu$ are the same - the degenerate linkages. Theorem 1.3 tells us that all the $a$ 's which are critical values for $\mu$ are rational multiples of $\pi$. Moreover, the smallest critical value is $2 \pi / n$ and the largest one is $(n-1) \pi / n$.

From the definition of $f, b$ and $w$ in Theorem 1.2, for $P \in M_{n}(a)$ a degenerate linkage, the following relations hold:

$$
\left\{\begin{array}{l}
f+b=n \\
a(f-b)=2 \pi w
\end{array}\right.
$$

Then we have the following:

Theorem 1.4. Let $P \in M_{n}(a)$ be a degenerate polygon. Then the following hold:

(i) When $w(P)>0$, the signature of $\left.D^{2} \mu\right|_{(P, a)}$ is given by

$$
(f(P)-2 w(P)-1, b(P)+2 w(P)-1) .
$$

(ii) When $w(P)<0$, the signature of $\left.D^{2} \mu\right|_{(P, a)}$ is given by

$$
(b(P)+2 w(P)-1, f(P)-2 w(P)-1) .
$$

This paper is organized as follows. In Section 2 we deduce several conclusions from Theorems 1.3 and 1.4. In particular, Theorem 2.1 is useful when we compute $\chi\left(M_{n}(a)\right)$. In Section 3 we prove Theorems 1.3 and 1.4. Theorem 1.2 is a key to proving Theorem 1.4. In Section 4 we give several examples about $\chi\left(M_{n}(a)\right)$.

\section{Acknowledgments}

The author is grateful to the referee for many thoughtful suggestions, in particular, for comments on the organization of the paper.

\section{Conclusions from Theorems 1.3 and 1.4}

In Theorem 1.4, we set

$$
(\alpha, \beta):= \begin{cases}(f(P)-b(P), 2 w(P)) & \text { if } w(P)>0 \\ (-f(P)+b(P),-2 w(P)) & \text { if } w(P)<0 .\end{cases}
$$

Then we obtain the following theorem. We denote by $\mathbb{N}=\{1,2,3, \ldots\}$ the set of natural numbers.

Theorem 2.1. We set

$$
\Gamma_{n}:=\{(\alpha, \beta) \in \mathbb{N} \times \mathbb{N} \mid \alpha \text { is odd, } \beta \text { is even and } \beta<\alpha \leqslant n\} .
$$

Then the following assertions hold:

(i) To each $(\alpha, \beta) \in \Gamma_{n}$, there corresponds a certain number of critical points of $\mu$. All critical points are non-degenerate such that their information is given by Table 1, where we set

$$
\alpha=2 s+1 \text { and } \beta=2 t
$$




\begin{tabular}{|c|c|c|}
\hline critical value & the number of critical points & the index \\
\hline$\frac{\beta}{\alpha} \pi$ & $\left(\begin{array}{c}n \\
m-s\end{array}\right)$ & $m-s+2 t-1$ \\
\hline
\end{tabular}

Table 1: The information on $\mu$ at $(\alpha, \beta)$

(ii) Conversely, a critical point of $\mu$ is attained by a unique $(\alpha, \beta) \in \Gamma_{n}$.

Remark 2.2. (i) It is not true that critical points of the same critical value have the same index. For example, $\Gamma_{9}$ contains elements $(3,2)$ and $(9,6)$ such that their critical values are $2 \pi / 3$. On the other hand, the index of the former is 4 but that of the latter is 5 .

(ii) The number of critical points does not depend on $\beta$. Moreover, the index of $\mu$ has the same parity as $m-s-1$, which also does not depend on $\beta$.

Proof of Theorem 2.1. (i) Let $U_{n}$ be the set of critical points of $\mu$. We define the map

$$
F: U_{n} \rightarrow \Gamma_{n}
$$

by making $(P, a) \in U_{n}$ correspond with $(\alpha, \beta)$ in (4). Note that by $(3), F$ is certainly a map to $\Gamma_{n}$.

We check Table 1 below. First, by (3) and (4), we have $a=\beta \pi / \alpha$ for $w$ positive or negative. Hence the critical value in Table 1 is true.

Second, we compute the index of $\mu$ at $(\alpha, \beta)$. Using (4) and (5), we have

$$
(f, b)= \begin{cases}(m+s+1, m-s), & \text { if } w>0, \\ (m-s, m+s+1), & \text { if } w<0\end{cases}
$$

and

$$
w= \begin{cases}t, & \text { if } w>0 \\ -t, & \text { if } w<0\end{cases}
$$

Using Theorem 1.4, (6) and (7), we have

$$
\text { the index of } \mu \text { at }(\alpha, \beta) \text { is } m-s+2 t-1
$$

for $w$ positive or negative. Hence the index of $\mu$ in Table 1 is true.

Third, we compute the number of critical points. (4) tells us that critical points $(P, a)$ and $(Q, a)$ satisfy $F(P, a)=F(Q, a)$ if and only if $(f(P), b(P))=(f(Q), b(Q))$ or $(f(P), b(P))=(b(Q), f(Q))$. The description of $f$ in terms of $s$ is computed in (6). Since $e_{n}$ is always back-track, we need to choose $f$-elements from $\left\{e_{1}, e_{2}, \ldots, e_{2 m}\right\}$. The total number of such choices is

$$
\left(\begin{array}{c}
2 m \\
m+s+1
\end{array}\right)+\left(\begin{array}{c}
2 m \\
m-s
\end{array}\right)=\left(\begin{array}{c}
2 m \\
m-s-1
\end{array}\right)+\left(\begin{array}{c}
2 m \\
m-s
\end{array}\right)=\left(\begin{array}{c}
2 m+1 \\
m-s
\end{array}\right)
$$

Hence the number of critical points in Table 1 is true. This completes the proof of Theorem 2.1 (i).

(ii) The item is clear from (4). 
Proposition 2.3. (i) As in the proof of Theorem 2.1, let $U_{n}$ be the set of critical points of $\mu$. Then we have

$$
\left|U_{n}\right|=\frac{1}{2}\left(-4^{m}+\frac{(2 m+1) !}{(m !)^{2}}\right)
$$

where $|-|$ stands for the cardinality.

(ii) Let $V_{n}$ be the set of critical values of $\mu$. We set

$$
\Phi(n):=\frac{1}{2} \sum_{s=1}^{m} \varphi(2 s+1),
$$

where $\varphi$ denotes Euler's totient function. Then we have $\left|V_{n}\right|=\Phi(n)$.

Proof. (i) We consider Table 1. If we fix $\alpha=2 s+1$, then the number of choices of $\beta$ is $s$. Moreover, if we fix $(\alpha, \beta)$, then the number of the corresponding critical points is $\left(\begin{array}{c}n \\ m-s\end{array}\right)$. Varying $s$ in $1 \leqslant s \leqslant m$, we have

$$
\left|U_{n}\right|=\sum_{s=1}^{m} s\left(\begin{array}{c}
n \\
m-s
\end{array}\right) .
$$

Using [14, A000531], we obtain (8).

(ii) We set

$$
\widetilde{\Gamma}_{n}:=\left\{(\alpha, \beta) \in \Gamma_{n} \mid \alpha \text { and } \beta \text { are coprime }\right\} .
$$

Table 1 tells us that

$$
\left|V_{n}\right|=\left|\widetilde{\Gamma}_{n}\right|
$$

Let $p_{1}: \widetilde{\Gamma}_{n} \rightarrow \mathbb{N}$ be the projection to the first factor. We claim that for each $\alpha=$ $2 s+1$, we have $\left|p_{1}^{-1}(\alpha)\right|=\varphi(\alpha) / 2$. In fact, if we forget the condition " $\beta$ is even" for $p_{1}^{-1}(\alpha)$, then its cardinality is $\varphi(\alpha)$. Moreover, since $\alpha$ is odd, exactly one of $\beta$ and $\alpha-\beta$ is even for all $\beta \in \mathbb{N}$. Namely, exactly one of $(\alpha, \beta)$ and $(\alpha, \alpha-\beta)$ is an element of $p_{1}^{-1}(\alpha)$. Hence the claim follows.

Now varying $s$ in $1 \leqslant s \leqslant m$, we have

$$
\left|\widetilde{\Gamma}_{n}\right|=\sum_{s=1}^{m} \varphi(2 s+1) / 2 .
$$

Combining (9) and (10), we obtain Proposition 2.3 (ii).

We study the asymptotic behavior of $\left|U_{n}\right|$ and $\left|V_{n}\right|$ in Proposition 2.3. In what follows, the notation

$$
f(n) \sim g(n) \quad(n \rightarrow \infty)
$$

means that

$$
\lim _{n \rightarrow \infty} \frac{f(n)}{g(n)}=1 .
$$

Corollary 2.4. We have the following results:

(i)

$$
\left|U_{n}\right| \sim 2^{2 m-1}\left(-1+\frac{2 m+1}{\sqrt{\pi m}}\right) \quad(m \rightarrow \infty) .
$$


(ii)

$$
\left|V_{n}\right| \sim \frac{n^{2}}{\pi^{2}} \quad(n \rightarrow \infty)
$$

Proof. (i) The item is a consequence of the following Stirling's formula:

$$
k ! \sim \sqrt{2 \pi k}\left(\frac{k}{e}\right)^{k} \quad(k \rightarrow \infty) .
$$

(ii) It will suffice to prove the following:

Lemma 2.5. We set

$$
\Psi(n):=\sum_{\substack{i=1 \\ i \text { odd }}}^{n} \varphi(i) .
$$

Then we have

$$
\Psi(n) \sim \frac{2 n^{2}}{\pi^{2}} \quad(n \rightarrow \infty)
$$

Proof of Lemma 2.5. To the best of the author's knowledge, there is no publication which proves the lemma. Hence we give a proof here. We modify the proof of $[\mathbf{3}$, Theorem 330], which claims that the average order of $\varphi(n)$ is $6 n / \pi^{2}$.

$$
\begin{aligned}
\Psi(n) & =\sum_{\substack{i=1 \\
i \text { odd }}}^{n} i \sum_{d \mid i} \frac{\mu(d)}{d}=\sum_{\substack{d d^{\prime} \leqslant n \\
d d^{\prime} \text { odd }}} d^{\prime} \mu(d) \\
& =\sum_{\substack{d=1 \\
d \text { odd }}}^{n} \mu(d) \sum_{\substack{d^{\prime}=1 \\
d^{\prime} \text { odd }}}^{\lfloor n / d\rfloor} d^{\prime}=\sum_{\substack{d=1 \\
d \text { odd }}}^{n} \mu(d)\left(\left\lfloor\frac{\lfloor n / d\rfloor-1}{2}\right\rfloor+1\right)^{2} \\
& \sim \frac{n^{2}}{4} \sum_{\substack{d=1 \\
d \text { odd }}}^{n} \frac{\mu(d)}{d^{2}} \sim \frac{n^{2}}{4} \sum_{\substack{d=1 \\
d \text { odd }}}^{\infty} \frac{\mu(d)}{d^{2}}=\frac{n^{2}}{4 \widetilde{\zeta}(2)}
\end{aligned}
$$

where we define

$$
\widetilde{\zeta}(2):=\sum_{\substack{j=1 \\ j \text { odd }}}^{\infty} \frac{1}{j^{2}}
$$

Since

$$
\zeta(2)=\widetilde{\zeta}(2)+\frac{1}{4} \zeta(2)
$$

we have

$$
\widetilde{\zeta}(2)=\frac{\pi^{2}}{8}
$$

Substituting (13) into (12), we obtain (11). This completes the proof of Lemma 2.5, and hence also that of Corollary 2.4 (ii). 


\section{Proofs of Theorems 1.3 and 1.4}

Proof of Theorem 1.3. We prove the theorem along the lines of [11, Theorem 2.9]. Combining the following three assertions, we obtain Theorem 1.3:

(i) (An analogue of $\left[\mathbf{1 1}\right.$, Lemma 2.7 (ii)].) By (2), the Zariski tangent space $T_{P} M_{n}(a)$ is given by

$$
T_{P} M_{n}(a)=\left.\operatorname{ker} d \mu\right|_{(P, a)} .
$$

(ii) (An analogue of $[\mathbf{1 1}$, Corollary 2.8].) We see from (i) that a point $P$ is a singular point of $M_{n}(a)$ if and only if $(P, a)$ is a critical point of $\mu$.

(iii) By $\left[\mathbf{1 0}\right.$, Theorem 1.1], $P$ is a singular point of $M_{n}(a)$ if and only if $P$ is degenerate.

In order to prove Theorem 1.4, we need the following:

Theorem 3.1. Let $h: M \rightarrow \mathbb{R}$ be a smooth function on a d-dimensional manifold $M$. For numbers $\xi_{1}<\xi_{2}$, we assume that $h^{-1}\left[\xi_{1}, \xi_{2}\right]$ is compact and contains a unique non-degenerate critical point $p$ of index $r$. Then the following results hold:

(i) The level set $h^{-1}\left(\xi_{1}\right)$ is obtained from $h^{-1}\left(\xi_{2}\right)$ by removing $\operatorname{Int}\left(\partial D^{d-r} \times D^{r}\right)$ and attaching $D^{d-r} \times \partial D^{r}$ along the boundary. We call this construction a surgery of type $(d-r, r)$.

(ii) If $d$ is odd, then we have

$$
\chi\left(h^{-1}\left(\xi_{1}\right)\right)=\chi\left(h^{-1}\left(\xi_{2}\right)\right)+2(-1)^{r+1} .
$$

(iii) We set $\xi_{3}:=h(p)$. Then level set $h^{-1}\left(\xi_{3}\right)$ is obtained from $h^{-1}\left(\xi_{2}\right)$ by removing Int $\left(\partial D^{d-r} \times D^{r}\right)$ and attaching $C\left(S^{d-r-1} \times S^{r-1}\right)$ along the boundary, where $C$ denotes the cone. In particular, if $d$ is odd, then we have

$$
\chi\left(h^{-1}\left(\xi_{3}\right)\right)=\chi\left(h^{-1}\left(\xi_{2}\right)\right)+(-1)^{r+1} .
$$

Proof. The theorem is well-known in Morse surgery. (See [13].)

Lemma 3.2. We consider the function $\rho_{n}$ in (1) for the case that $r^{\prime}=(a, a, \ldots, a)$, that is, $r_{i}=a$ for $1 \leqslant i \leqslant n-1$. Let $O$ be an open neighborhood of $(P, a)$ in $X_{n}$ such that $O$ contains no other degenerate polygons than $(P, a)$. We fix a sufficiently small positive real number $\varepsilon$. Then the following assertions hold:

(i) Assume that $w(P)>0$. Then for all $j \in\{-1,1\}, \mu^{-1}(a+j \varepsilon) \cap O$ is diffeomorphic to an open set of $\rho_{n}^{-1}(a-j \varepsilon)$.

(ii) Assume that $w(P)<0$. Then for all $j \in\{-1,1\}, \mu^{-1}(a+j \varepsilon) \cap O$ is diffeomorphic to an open set of $\rho_{n}^{-1}(a+j \varepsilon)$.

Proof of Lemma 3.2. (i) We write the side-lengths of a spherical polygon as $\left(r_{1}, r_{2}, \ldots\right.$ $\left.\ldots, r_{n}\right)$. For $\delta \in[0, \varepsilon]$, we deform the side-lengths of an element of $\mu^{-1}(a+\varepsilon) \cap O$ by

$$
(a+j \delta, a+j \delta, \ldots, a+j \delta, a+j(-\varepsilon+2 \delta)) .
$$

We need to check that this deformation is indeed possible. To see this, it will suffice to see that (14) does not cross a wall for any $\delta$, where a wall is defined in [11, p. 311]. 
Recall that $e_{n}$ is always back-track. (See Definition 1.1.) Hence we have about (14) that

the sum of forward-track side-lengths - the sum of back-track side-lengths

$$
=2 \pi w(P)+j(\varepsilon+(u-2) \delta)
$$

for some integer $u$. We claim that $u \geqslant 2$. In fact, the assumption $w(P)>0$ tells us that at least $m+1$ of the first $2 m$ components of (14) are forward-track. Hence we have $u \geqslant(m+1)-(m-1)=2$ and the claim follows.

Now since the term $j(\varepsilon+(u-2) \delta)$ in (15) is near zero but not zero for all $\delta \in[0, \varepsilon]$, (14) does not cross a wall for any $\delta$. Finally, setting $\delta=0$ or $\varepsilon$ in (14), we complete the proof of (i).

(ii) Instead of (14), we deform the side-lengths of an element of $\mu^{-1}(a+\varepsilon) \cap O$ by

$$
(a+j \delta, a+j \delta, \ldots, a+j \delta, a+j \varepsilon) .
$$

We have about (16) that

the sum of forward-track side-lengths - the sum of back-track side-lengths

$$
=2 \pi w(P)-j(\varepsilon+v \delta)
$$

for some integer $v$. We claim that $v \geqslant 0$. In fact, the assumption $w(P)<0$ tells us that at most $m$ of the first $2 m$ components of (16) are forward-track. Hence we have $-v \leqslant m-m=0$ and the claim follows.

Now since the term $j(\varepsilon+v \delta)$ in $(17)$ is near zero but not zero for all $\delta \in[0, \varepsilon]$, (16) does not cross a wall for any $\delta$. Finally, setting $\delta=0$ or $\varepsilon$ in (16), we complete the proof of (ii). This completes the proof of Lemma 3.2.

Proof of Theorem 1.4. We shall deduce Theorem 1.4 from Theorem 1.2. We apply Theorem 3.1 (i) to the map $\mu$. Note that $\operatorname{dim} X_{n}=n-2$. We denote by $r$ the index of $P$. When the level set $\mu^{-1}(a+\varepsilon)$ descends to $\mu^{-1}(a-\varepsilon)$, the critical point $P$ gives a surgery of type

$$
(n-2-r, r)
$$

We set $\lambda:=f(P)-2 w(P)-1$.

(i) If $w(P)>0$, then Lemma 3.2 (i) tells us that the above descent is equivalent to the ascent from $\rho_{n}^{-1}(a-\varepsilon)$ to $\rho_{n}^{-1}(a+\varepsilon)$. Combining Theorem 3.1 (i) and Theorem 1.2 , when we cross through $P$, a surgery of type

$$
(\lambda, n-2-\lambda)
$$

occurs. Comparing (18) and (19), we have

$$
r=n-2-\lambda=b(P)+2 w(P)-1 .
$$

(ii) If $w(P)<0$, then the descent from $\mu^{-1}(a+\varepsilon)$ to $\mu^{-1}(a-\varepsilon)$ is equivalent to the descent from $\rho_{n}^{-1}(a+\varepsilon)$ to $\rho_{n}^{-1}(a-\varepsilon)$. When we cross through $P$, a surgery of type

$$
(n-2-\lambda, \lambda)
$$

occurs. Comparing (18) and (20), we have

$$
r=\lambda=f(P)-2 w(P)-1 .
$$

This completes the proof of Theorem 1.4. 


\section{Examples}

Combining Theorems 2.1 and 3.1, we have a procedure to compute $\chi\left(M_{n}(a)\right)$. For example, if we are in one of the following cases, we can obtain explicit formula for $\chi\left(M_{n}(a)\right)$ :

(i) The case that $n$ is not too big and all $a$.

(ii) The case that $n$ is any odd number and $a$ is near the smallest or largest critical value of $\mu$.

As illustrative examples, we prove the following:

Proposition 4.1. (i) For all odd numbers n, we have

$$
\chi\left(M_{n}(\pi / 2)\right)=(-1)^{m+1} \cdot 2^{2 m-1} .
$$

(ii) If $0<a<2 \pi / n$, then we have

$$
\chi\left(M_{n}(a)\right)=(-1)^{m+1}\left(\begin{array}{c}
2 m \\
m
\end{array}\right) .
$$

Recall that $2 \pi / n$ is the smallest critical value of $\mu$.

Proof. (i) We set

$$
S:=\left\{\frac{2 t}{2 s+1} \pi \mid 1 \leqslant s \leqslant m,\lfloor s / 2\rfloor+1 \leqslant t \leqslant s\right\} .
$$

It is clear that $\zeta \in V_{n}$ satisfies $\pi / 2<\zeta$ if and only if $\zeta \in S$. Using Theorem 2.1, Remark 2.2 (ii), Theorem 3.1 (ii) and (21), we have

$$
\begin{aligned}
\chi\left(M_{n}(\pi / 2)\right) & =2 \sum_{s=1}^{m}(-1)^{m+s}(s-\lfloor s / 2\rfloor)\left(\begin{array}{c}
n \\
m-s
\end{array}\right) \\
& =2(-1)^{m} \sum_{s=1}^{m}(-1)^{s}\lfloor(s+1) / 2\rfloor\left(\begin{array}{c}
2 m+1 \\
m-s
\end{array}\right) \\
& =2(-1)^{m+1} \sum_{i=0}^{m+1}(-1)^{i}\lfloor i / 2\rfloor\left(\begin{array}{c}
2 m+1 \\
m+i
\end{array}\right) .
\end{aligned}
$$

It is proved in $[\mathbf{7}]$ that

$$
\sum_{i=0}^{m+1}(-1)^{i}\lfloor i / 2\rfloor\left(\begin{array}{c}
2 m+1 \\
m+i
\end{array}\right)=2^{2 m-2} .
$$

Hence we have from $(22)$ that $\chi\left(M_{n}(\pi / 2)\right)=(-1)^{m+1} \cdot 2^{2 m-1}$.

(ii) Similarly to (i), we have

$$
\chi\left(M_{n}(a)\right)=2 \sum_{s=1}^{m}(-1)^{m+s} s\left(\begin{array}{c}
n \\
m-s
\end{array}\right)=(-1)^{m+1}\left(\begin{array}{c}
2 m \\
m
\end{array}\right) .
$$

Remark 4.2. (i) Proposition 4.1 (i) was obtained in [6, Theorem 2] by a different method. 
(ii) The space $M_{n}(a)$ in Proposition 4.1 (ii) is diffeomorphic to the equilateral polygon space in $\mathbb{R}^{2}$. The formula for $\chi\left(M_{n}(a)\right)$ is in agreement with the one obtained in $[\mathbf{5}$, Theorem A].

\section{References}

[1] M. Farber, Invitation to Topological Robotics, Zurich Lectures in Advanced Mathematics, European Mathematical Society (EMS), 2008.

[2] M. Farber and D. Schütz, Homology of planar polygon spaces, Geom. Dedicata 125 (2007), 75-92.

[3] G.H. Hardy and E.M. Wright, An Introduction to the Theory of Numbers, 6th ed., Oxford University Press, 2008.

[4] J.-C. Hausmann, Sur la topologie des bras articulés, Lecture Notes in Mathematics 1474, Springer-Verlag, Berlin, 1989, pp. 146-159.

[5] Y. Kamiyama, Topology of equilateral polygon linkages, Topology Appl. 68 (1996), 13-31.

[6] Y. Kamiyama, A hypersurface of the configuration space of a spatial robot arm, JP J. Geom. Topol. 20 (2017), 27-38.

[7] Y. Kamiyama, Sum of terms involving binomial coefficients and floor functions. Available at https://math.stackexchange.com/questions/2636484 [2018, 5, February].

[8] M. Kapovich and J. Millson, On the moduli space of polygons in the Euclidean plane, J. Differential Geom. 42 (1995), 430-464.

[9] M. Kapovich and J. Millson, The symplectic geometry of polygons in Euclidean space, J. Differential Geom. 44 (1996), 479-513.

[10] M. Kapovich and J. Millson, Hodge theory and the art of paper folding, Publ. Res. Inst. Math. Sci. 33 (1997), 1-33.

[11] M. Kapovich and J. Millson, On the moduli space of a spherical polygonal linkage, Canad. Math. Bull. 42 (1999), 307-320.

[12] R.J. Milgram and J.C. Trinkle, The geometry of configuration spaces for closed chains in two and three dimensions, Homology Homotopy Appl. 6 (2004), 237267.

[13] J. Milnor, Morse Theory, Annals of Mathematics Studies 51, Princeton Univ. Press, Princeton, N.J., 1963.

[14] N.J.A. Sloane, The On-Line Encyclopedia of Integer Sequences. Available at http://oeis.org.

[15] K. Walker, Configuration Spaces of Linkages, Undergraduate thesis, Princeton, 1985 .

Yasuhiko Kamiyama kamiyama@sci.u-ryukyu.ac.jp

Department of Mathematics, University of the Ryukyus, Nishihara-Cho, Okinawa 903-0213, Japan 